\title{
Uso y funciones de Twitter en periodistas españoles
}

\author{
Antonio RoDRÍGUEZ RUIBAL \\ Universidad a distancia de Madrid (UDIMA) \\ antonio.rodriguez@udima.es \\ Javier GARCÍA LÓPEZ \\ Universidad a Distancia de Madrid (UDIMA) \\ javier.garcia@udima.es
}

Recibido: $11 / 10 / 2012$

Aceptado: 23/01/2013

\begin{abstract}
Resumen
El uso de las redes sociales en internet, ha hecho que las fuentes y los canales informativos estén cambiando. Los periodistas de los principales medios de comunicación españoles, han empezado a utilizar muy proactivamente destacadas redes sociales, en especial la red de microbbloging Twitter. La firma de sus escritos generalmente en géneros periodísticos de opinión, va acompañada en ocasiones por su nick de esta red social, lo que provoca que se convierta en escaparate publicitario de esta red social y de su propia marca personal. La celeridad en la actualización de esta red social digital y su limitación a 140 caracteres, provoca que la información y los titulares sean constantes e inmediatos. Pero no todo es información ¿Quién debe dar la noticia, el periodista o el medio?
\end{abstract}

Palabras clave: Twitter, redes sociales, periodista

\section{Spanish Journalists: Use and Functions of Twitter}

\begin{abstract}
The use of social networking sites has made the sources and channels of information are changing. Journalists from leading Spanish media, have started using very proactively leading social networks, especially Twitter microbbloging network. The signing of journalistic genres usually written opinion, sometimes accompanied by his nickname of this social network, causing it to become advertising showcase this social network and your own personal brand. The speed in updating this digital social network and its limitation to 140 characters, causing information and headlines are constant and immediate. But not all information Who should deliver the news, journalist or media?
\end{abstract}

Keywords: Twitter, Social Media, Journalist

\section{Referencia normalizada}

RODRÍGUEZ RUIBAL, Antonio y GARCÍA LÓPEZ, Javier (2013): “Uso y funciones de Twitter en periodistas españoles”. Estudios sobre el mensaje periodístico. Vol. 19. Núm. especial abril, págs.: 963969. Madrid, Servicio de Publicaciones de la Universidad Complutense.

Sumario: 1. Introducción. 2. Metodología. 3. Desarrollo. 4. Conclusiones. 5. Referencias bibliográficas.

\section{Introducción}

El uso de las redes sociales en internet, ha hecho que las fuentes y los canales informativos estén cambiando. Los periodistas de los principales medios de comunicación españoles, han empezado a utilizar muy proactivamente destacadas redes sociales, en especial la red de microbbloging Twitter. La firma de sus escritos generalmente en géneros periodísticos de opinión, va acompañada en ocasiones por su nick de esta red social, lo que provoca que se convierta en escaparate publicitario de esta red social y de su propia marca personal. 
La celeridad en la actualización de esta red social digital y su limitación a 140 caracteres, provoca que la información y los titulares sean constantes e inmediatos. Pero no todo es información ¿Quién debe dar la noticia, el periodista o el medio? En este proceso de adaptación periodística de los medios de comunicación hacia las redes sociales, en este trabajo de investigación se analiza el uso y los contenidos que los periodistas españoles más activos en cuanto a número de seguidores y de aportaciones (o tweets), hacen e incluyen en sus perfiles de Twitter.

Las aportaciones de los periodistas españoles en Twitter, no sólo es informativa, sino que gran parte de las mismas son conversaciones con usuarios, rebote de información (o retweets), información sobre gustos personales de toda índole o reflexiones personales sobre temas ajenos a la profesión periodística. ¿Cuentan los periodistas con su medio? ¿los medios cuentan, apoyan y conocen la actividad de sus periodistas?

Moda pasajera o no, los medios de comunicación han tenido que adaptarse a la instantaneidad y la viralidad que caracteriza a este microbbloging. Movimientos sociales como el de la primavera árabe, el $15 \mathrm{M}$ o la reciente huelga minera han hecho que, tanto las empresas editoriales como los propios periodistas, quieran estar presentes en estas redes y en especial Twitter, para estar informados e informar, con mayor o menor éxito.

Sin duda alguna, este fenómeno no tan nuevo en cuanto a contexto sino quizás a la forma, ha provocado un daño directo en la manera de informarse, y por lo tanto de hacer periodismo. Cada una de las aportaciones que se hacen en Twitter está compuesta como máximo por 140 caracteres, lo que provoca que cada pequeña frase deba ser un titular. Los usuarios tienen que leer/consumir miles de tweets, y hay que llamar la atención de los seguidores y conseguir una máxima difusión a través de retweets o RT.

Profundizar queda entonces en un segundo plano. La falta de vínculos a otras páginas web en donde se amplíe la información, se añada contenido multimedia o se informe con detalle de lo ocurrido, es cada vez más importante. Una frase tan corta no supone un atractivo para los usuarios de esta red social, salvo excepciones.

Con el paso del tiempo y el continuo proceso de aprendizaje y adaptación de la red social y de los usuarios a la misma, Twitter ha ido añadiendo la posibilidad de compartir otra serie de elementos como son la localización geográfica, fotografías o ha generalizado el uso del carácter \# (almohadilla) seguido de una palabra que resuma el contenido, que toma el término de hashtag. De esta forma, todos los usuarios pueden seguir una temática global siempre que incluyan el mismo término. Además, gracias al acortamiento automático de las enlaces por parte de Twitter, los usuarios cuentan con más espacio para incluir vínculos externos y ampliar la información.

José Miguel Flores (2009) dice "Ante este panorama, el periodista debe ser, a su vez, participativo. Es decir, hay que buscar y encontrar el tiempo para los comentarios publicados como consecuencia de las noticias, de las historias y escribir sobre aquéllos que merezcan ser respondidos. Hay que ser proactivo en buscar retroalimentación sobre las historias antes de que sean publicadas. Esa proactividad puede ser tan simple como publicar un "llamado a los lectores" en el periódico, o tan avanzado como configurar una lista de correo electrónico con destinatarios seleccionados. Por ejemplo, si un periodista cubre el área de educación, debería construir una lista -de profesores e investigadores y directivos relacionados con el sector- con el objetivo de 
enviarles ráfagas de correos electrónicos cuando se necesite de comentarios generales para una determinada historia. Este es el preámbulo para establecer una red en este sector." Ahora bien, ¿se ha convertido el periodismo en un lanzamiento de cibertitulares por parte de medios de comunicación y periodistas? ¿Cuál es el objetivo de los medios en esta red social: tener más seguidores, dar más titulares, tener más tráfico en su página web para que así les surjan oportunidades comerciales? ¿Quién es el encargado de dar la noticia, el medio o el periodista? ¿Tienen protocolos de actuación los medios para sus trabajadores?

Muchas de esas preguntas no tienen una respuesta científica ni se puede cuantificar. Sin embargo algunos medios de comunicación de referencia como el New York Times o la BBC, ya cuentan con códigos internos de comportamiento en redes sociales, que regulan y ponen límite al uso de las redes sociales en el trabajo. Es tal la repercusión que muchos periodistas cuentan con más seguidores y aportaciones que el medio de comunicación que les paga.

\section{Metodología}

Para usar Twitter como medio de estudiar la popularidad o sentimientos de los usuarios (Rojo: 2012: 12-13) hacia un producto, marca o servicio, se puede medir -entre otros factores- la cantidad de veces que ese elemento fue mencionado en la red social. Dada la naturaleza de esta red pueden existir muchas formas en que las personas se refieren al elemento a medir (en el extremo, tantas formas como emisores posibles) por lo que es necesario realizar un proceso de recolección de "palabras claves" que las personas utilizan al momento de referirse al producto o servicio, las que pueden variar desde el nombre del elemento, marcas o personajes asociados.

En particular como cita Rojo Pizarro, los usuarios han utilizado una forma especial de palabras claves definida durante la escritura del mensaje llamada Hashtag, ésta consiste en escribir el símbolo \# seguido de una cadena concatenada de texto que hace referencia a un evento, hecho o situación. El uso del hashtag es una convención creada orgánicamente por la comunidad de usuarios para agregar mayor nivel de contexto y agregar metadata a las conversaciones en Twitter u otras redes. Es importante destacar que el hashtag no hace distinción ente mayúsculas y minúsculas (e.g. es lo mismo decir \#HashTag que \#hashtag).

Con la información recolectada sobre un conjunto de palabras claves en Twitter se pueden realizar diversas mediciones como son:

- Popularidad: cantidad de tweets sobre un tópico en un tiempo determinado.

- Sentimiento / Emoción: análisis de los contenidos de un tweet, en tanto son una apreciación positiva, neutral o negativa sobre un tópico en un tiempo determinado. Existen diversos medios para realizar este análisis, desde el método manual (donde el investigador cataloga cada tweet como positivo, neutro o negativo) hasta algoritmos avanzados basados en métodos de minería de datos y en análisis semántico.

- Influencia: cuáles y cuántos son los usuarios más influyentes que hablaron sobre un tópico en un tiempo determinado, existen varias medidas de influencia como son cantidad de seguidores, de retweets, o de menciones sobre un usuario. 
No existe una lista oficial de periodistas españoles que utilicen Twitter, pero si alguna realizada en el contexto de esa red social. Para el estudio, se va a tomar como referencia al Profesor de la Universidad de Navarra, D. José Luis Orihuela (a través de su perfil denominado @jlori), que tiene publicado en el momento de hacer la investigación (septiembre de 2012) una lista bajo el nombre "Spanish journalist". Actualmente cuenta con 360 periodistas que utilizan esta red social de manera cotidiana.

\section{Desarrollo}

Se van a tener en cuenta para el análisis del uso de Twitter por parte de estos periodistas, a los seguidores (o followers), las listas (en las que el usuario está inscrito voluntaria o involuntariamente) y los tweets aportados.

En cuanto al primer ítem, el ranking de los veinte primeros queda así (a día 24 de septiembre de 2012):

\begin{tabular}{|l|c|c|c|}
\hline Usuario & Followers & Listas & Tweets \\
\hline _anapastor_ & 432609 & 7969 & 19768 \\
\hline maldinisport & 328388 & 5398 & 19575 \\
\hline juanmacastano & 195286 & 3577 & 12827 \\
\hline iescolar & 175415 & 6616 & 24259 \\
\hline julia_otero & 147855 & 2998 & 13909 \\
\hline jlori & 147779 & 4226 & 16452 \\
\hline pedroj_ramirez & 119908 & 3713 & 31899 \\
\hline marilink & 115706 & 2362 & 29172 \\
\hline JavierCapitan & 93132 & 910 & 3317 \\
\hline MonicaCarrillo & 83492 & 895 & 3724 \\
\hline VicenteVallesTV & 83290 & 2576 & 3014 \\
\hline carlos_alsina & 54553 & 1485 & 5927 \\
\hline melchormiralles & 53241 & 1954 & 79243 \\
\hline anablanco_tve & 44994 & 1100 & 204 \\
\hline javierares & 41353 & 1396 & 3861 \\
\hline carlosecue & 34376 & 1247 & 6301 \\
\hline radiocable & 33998 & 1508 & 18384 \\
\hline VictorAmela & 33755 & 599 & 4533 \\
\hline Evature & 32669 & 448 & 6601 \\
\hline Noemidemiguel & 31925 & 717 & 10672 \\
\hline
\end{tabular}

Como se puede observar, las diferencias entre el primero y el último usuario de este listado es muy grande, casi diez veces más entre la periodista Ana Pastor @_anapastor_y Noemí de Miguel @Noemidemiguel. Pero el número de seguidores no implica nada en especial más allá del interés popular o profesional de ese usuario.

Cabe destacar dos apartados muy interesantes que vinculan la especialización, el interés y la identificación del periodista con su propio medio, es decir, si nos fijamos en los tres primeros, podemos ver que tanto el segundo como el tercer nick se refieren a Maldini y Juanma Castaño, periodistas deportivos. Esta especialización del pe- 
riodismo tiene una gran aceptación entre los usuarios de la red social. En cuanto al sentimiento de pertenencia de los periodistas con su medio, desconociendo las políticas internas de los mismos, cabe destacar que sólo la periodista Ana Blanco, utiliza el nombre de su empresa en su nombre de usuario @anablanco_tve. El resto de los veinte que tienen más seguidores de los 360 usuarios de la lista "spanish journalist", ninguno repite esa fórmula.

Otra de las grandes cuestiones es saber la participación de los periodistas, cabría resolver la siguiente pregunta: ¿qué aportan estos veinte periodistas en Twitter?

Los usuarios de las redes sociales se ven necesariamente a utilizar 3 tipos de contenidos:

»Contenido o actualización de tipo profesional

»Contenido multimedia o información de otros medios

» Contenido personal

Si a esos tres contenidos, le añadimos otra variable, que sea el uso de información de su propio medio, obtenemos un cuadro que está compuesto por: contenido informativo (I), contenido de información personal (P), información que vincula a su medio (M) y rebote de información de otro usuario o retweet (RT).

El cuadro a 01 de octubre de 2012 queda compuesto ahora de la siguiente manera:

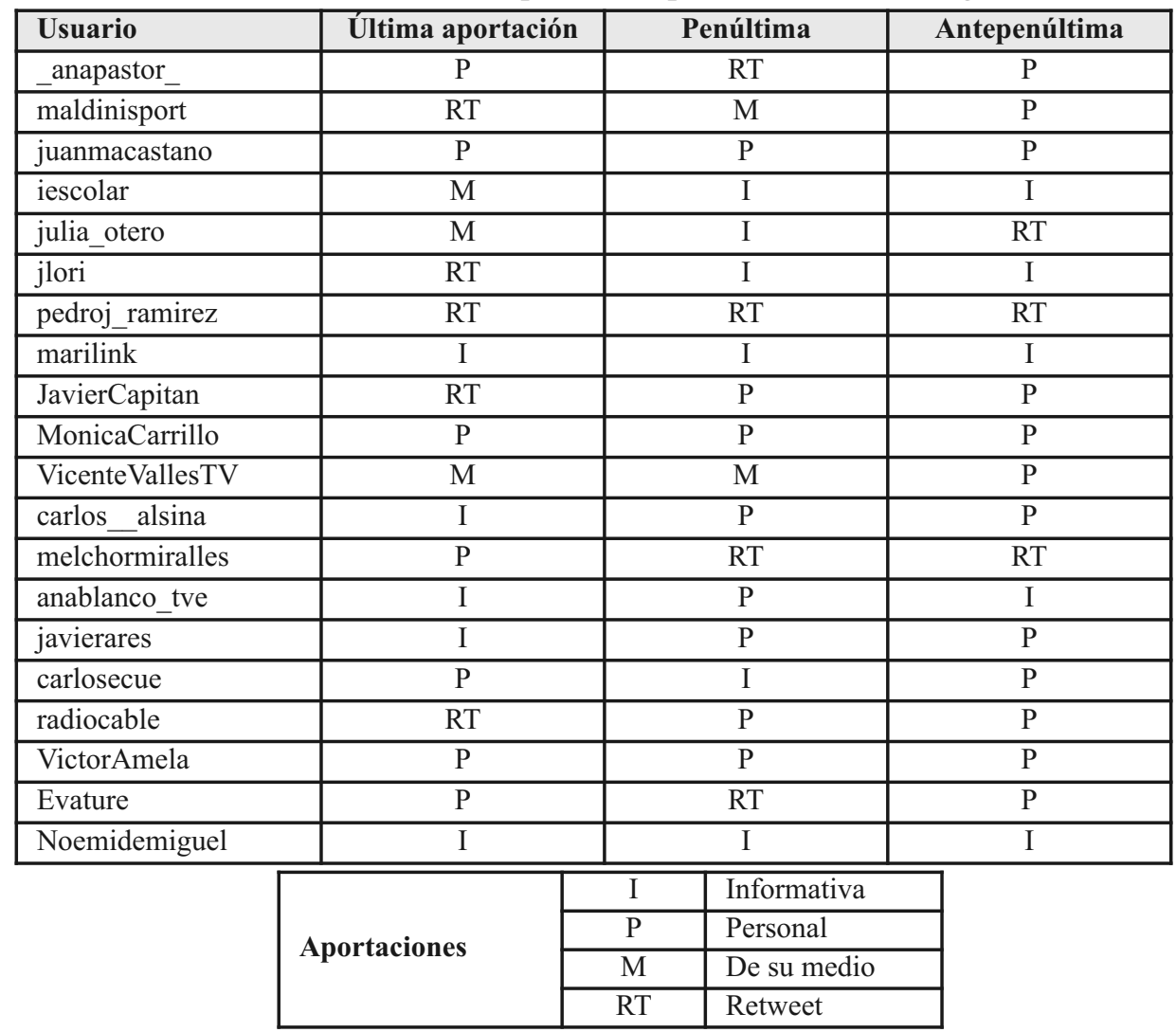


El ranking varía notablemente en cuanto a número de aportaciones de los periodistas en la red social. Tener más seguidores no depende, como queda demostrado, del número de aportaciones. Según Tíscar Lara (2008): "Los medios, si quieren atraer y consolidar redes sociales con y entre sus audiencias, deben incidir en aspectos como la conectividad, el servicio, la participación abierta y de calidad, la orientación y dinamización y la gestión del conocimiento."

\section{Conclusiones}

El contenido de las redes sociales de los periodistas analizados muestra el diferente uso y aceptación que tiene entre ellos. Aunque no era motivo de la investigación, es interesante analizar la firma de todos ellos, en donde prácticamente ninguno lo vincula a su medio de comunicación, y cuando lo hacen nunca al perfil de su institución en esta red social. De la misma manera, aunque no se ha estudiado, sería interesante confirmar que la gran mayoría de ellos utilizan a sus medios para promocionar sus perfiles y conseguir así más followers y porqué no, un mejor posicionamiento online.

Esto demuestra que no hay una vinculación entre periodista-medio o no parece que por haya una obligación o unas pautas por parte del medio de comunicación. Sólo el $8,3 \%$ del total de los tweet analizados, hacen vinculación a su medio de comunicación.

En cuanto al tipo de contenido, aunque los usuarios son muy activos ya que todo ellos habían publicado una hora antes del análisis, el $45 \%$ de los mismos introdujo comentarios de tipo personal en su time list, lo que demuestra que el uso profesional queda a un lado, aunque lo promocionen en sus diferentes medios de comunicación.

E1 20\% de las aportaciones, vienen derivados de otros usuarios, es decir, son retweet de otros, lo que supone además, que los periodistas no sólo son fuentes de información, sino soportes informativos en esta red social.

Sólo, el 26,7\% de los comentarios de los periodistas en Twitter son de carácter meramente informativo, lo que apoya a la teoría de lo ya descrito con anterioridad, es decir, si el uso de Twitter tiene fines personales o de ocio, en vez de informativos aún cuando promocionan su usuario a través de sus medios de comunicación.

\section{Referencias bibliográficas}

CAMPOS FREIRE, Francisco (2010): "Las redes sociales trastocan los modelos de los medios de comunicación tradicionales", en Revista Latina de comunicación $\mathrm{n}^{\circ}$ 63. Disponible en: www.ull.es/publicaciones(latina/_2008/23_34/Santiago/Francisco_Campos.htm. [Consultado: 20 de septiembre de 2012]

CERVERA FANTONI, Ángel Luis (2009): Comunicación total. Madrid, ESIC.

FLORES VIVAR, Jesús Miguel (2009): "Nuevos modelos de comunicación, perfiles y tendencias en las redes sociales", en Comunicar Revista Científica de Educomunicación, $\mathrm{n}^{\mathrm{o}} 33$. Disponible en: www.revistacomunicac.om/verpdf.php?numero=33-2009-09. [Consultado: 20 de septiembre de 2012]

LARA, Tiscar (2008): "La nueva esfera pública. Los medios de comunicación como redes sociales", en Revista TELOS, $\mathrm{n}^{\mathrm{o}} 76$. Disponible en: http://sociedadinforma- 
cion.fundacion.telefonica.com/telos/articulocuaderno.asp@idarticulo\%3D9\&rev\% 3D76.htm [Consultado: 18 de septiembre de 2012]

NOGUERA VIVO, José Manuel (2010): "Redes sociales como paradigma periodístico. Medios españoles en Facebook", en Revista de Comunicación Latina $\mathrm{n}^{\circ} 65$. Disponible en: www.revistalatinacs.org/10/art/891_UCAM/13:JM_Noguera.html ROJO PIZARRO, Rodrigo (2012): "Existe alguna relación entre la audiencia televisiva y la cantidad de tweets sobre un programa: el caso de Mundos Opuestos". Santiago de Chile. Facultad de Economía Negocios. Universidad de Chile

\section{Antonio RODRÍGUEZ RUIBAL}

Universidad a Distancia de Madrid (UDIMA)

Profesor del Grado en Periodismo

antonio.rodriguez@udima.es

\section{Javier GARCÍA LÓPEZ}

Universidad a Distancia de Madrid (UDIMA)

Profesor del Grado en Periodismo

javier.garcia@udima.es 